\title{
Plasma and PBMC miRNA Profile in Sexually HIV-1Exposed Seronegative Individuals
}

Mara Biasin ${ }^{1}$, Sara Yahyaei ${ }^{1}$, Mariacristina De Luca ${ }^{1}$, Irma Saulle ${ }^{1}$, Federica Gnudi ${ }^{1}$, Salome Ibba $^{1}$, Micaela Garziano $^{1}$, Angela Berzi ${ }^{1}$, Veronica Rainone1, Daria Trabattoni ${ }^{1}$, Sergio Lo Caputo ${ }^{2}$, Francesco Mazzotta ${ }^{2}$, Mario Clerici ${ }^{3}$

${ }^{1}$ University of Milan, Biomedical and Clinical Sciences, Milan,Italy, ${ }^{2}$ S. Maria Annunziata Hospital, Florence, Italy, ${ }^{3}$ Don Gnocchi Foundation, Milan, Italy

Background: MicroRNAs (miRNAs) are small 20- to 24-nt non-coding RNAs involved in the posttranscriptional regulation of gene expression which play important defensive roles in several viral infections. Global expression profiles of cellular miRNAs have identified alterations of specific miRNAs post-HIV-1 infection both in vitro and in different patient cohorts suggesting potential roles for miRNA in pathogenesis and disease progression. We therefore decided to verify if natural resistance to HIV-1 infection observed in seronegative individuals repeatedly exposed to HIV-1 (HESN) through unprotected sexual intercourse could be secondary to a different expression of their miRNA profile.

Methods: Expression levels of 25 miRNAs selected according to their proven anti-HIV-1 properties were analyzed in plasma,basal PBMC and in in vitro HIV-1 infected macrophages isolated from 30 HESN, 30 HIV seropositive subjects (HIV + ) and 30 healthy controls (HC).

Results: In plasma the expression of mir-155, mir-382, mir-28 and mir-198 was significantly augmented in both HIV + and HESN compared to HC probably as a consequence of viral exposure. Conversely the expression of mir-223 and mir-150 in plasma was significantly increased only in HESN and this result was also confirmed in basal PBMC suggesting a protective effect for these miRNAs in resistance to HIV-1 infection. Furthermore, the expression of mir-150 was significantly increased in HESN macrophages following HIV-1 infection.

Conclusions: mir-223 and mir-150 can target the $3 \not$ UTR of HIV -1 transcripts, and they have already been identified as anti-HIV-1 miRNAs. The higher expression of these miRNA in HESN samples could therefore represent a key protection mechanism against HIV infection. 